Research Paper

\title{
Nitrogen Containing Bisphosphonates Impair the Release of Bone Homeostasis Mediators and Matrix Production by Human Primary Pre-Osteoblasts
}

\author{
Chiara Giannasi 1,2, Stefania Niada 2, Davide Farronato ${ }^{3}$, Giovanni Lombardi 2, Barbara Manfredi ${ }^{1}$, \\ Giampietro Farronato ${ }^{1,4}$ and Anna Teresa Brini 1,2 $₫$ \\ 1. Department of Biomedical, Surgical and Dental Sciences, University of Milan, Milan, Italy. \\ 2. IRCCS Istituto Ortopedico Galeazzi, Milan, Italy. \\ 3. Department of Medicine and Surgery, Insubria University, Varese, Italy. \\ 4. IRCCS Fondazione Ca' Granda Ospedale Maggiore Policlinico, Milan, Italy. \\ $\square$ Corresponding author: Anna Teresa Brini, Department of Biomedical, Surgical and Dental Sciences, University of Milan, via Vanvitelli 32, 20129 Milan, Italy. \\ Tel: +39-02-50316988; Fax: +39-02-50316987; E-mail: anna.brini@unimi.it. \\ (c) Ivyspring International Publisher. This is an open access article distributed under the terms of the Creative Commons Attribution (CC BY-NC) license \\ (https://creativecommons.org/licenses/by-nc/4.0/). See http://ivyspring.com/terms for full terms and conditions.
}

Received: 2018.05.25; Accepted: 2018.10.11; Published: 2019.01.01

\begin{abstract}
Bisphosphonates (BPs) represent the first-line treatment for a wide array of bone disorders. Despite their well-known action on osteoclasts, the effects they induce on osteoblasts are still unclear. In order to shed light on this aspect we evaluated the impact of two nitrogen containing bisphosphonates, Alendronate (ALN) and Zoledronate (ZOL), on human primary pre-osteoblasts. At first, we showed an inhibitory effect on cell viability and alkaline phosphatase activity starting from $\mu \mathrm{M}$ concentrations of both drugs. In addition, an inhibitory trend on mineralized nodules deposition was observed. Then low doses of both ALN and ZOL rapidly increased the release of the pro-inflammatory mediators TNF $\alpha$ and IL-1 $\beta$, while increased DKK-1 and Sclerostin, both inhibitors of osteoblastogenesis. Finally, ALN and $10^{-7} \mathrm{M}$ ZOL decreased the expression of type I Collagen and Osteopontin, while both drugs slightly stimulated SPARC production. With these results, we would like to suggest a direct inhibitory action on bone-forming cells by nitrogen containing bisphosphonates.
\end{abstract}

Key words: bisphosphonates; human primary pre-osteoblasts; bone formation

\section{Introduction}

Bisphosphonates (BPs) represent the elective therapy for several metabolic and oncological diseases affecting the skeletal system, such as different types of osteoporosis, Paget disease, osteogenesis imperfecta, fibrous dysplasia and primary or secondary bone cancer. Among nitrogen containing bisphosphonates, Alendronate (ALN) is mainly used for the prevention and treatment of osteoporosis (10mg once a day or 70mg once a week, per os administration). Conversely, Zoledronate $(\mathrm{ZOL})$, one of the most potent nitrogen containing BPs, is usually intravenously injected in patients with advanced malignancies to prevent skeletal complications, such as pathological fractures, cancer-induced bone loss or hypercalcemia (posology:
$4 \mathrm{mg}$ every 3 to 4 weeks), or in the treatment of osteoporosis (5mg once a year). Despite BPs' well-known therapeutic potential, they also present important side effects. In particular, prolonged treatment with these drugs seems to predispose to the development of paradoxical side effects affecting bone, such as osteonecrosis of the jaw (Bisphosphonate Related Osteonecrosis of the Jaw, BRONJ) and atypical femoral fractures (AFFs) $[1,2]$. The pathophysiology of these skeletal conditions is still under investigation, as their etiology seems to depend on the synergy of several factors [3]. For both side effects, drug-induced suppression of bone turnover, resulting in an impairment of bone quality 
and architecture, has been identified as the starting cause. For BRONJ, BP anti-angiogenic action, inflammation, genetic predisposition and altered immune status are recognized among the main causes and favoring factors [4]. For AFFs, changes in mineral density and/or distribution, together with micro damage accumulation, are listed [3]. Recently it has been estimated that the frequency of BRONJ onset in oncology patients receiving high doses of BPs spans from 1 to $15 \%$, while in osteoporosis patient its prevalence is not enhanced $(0.001 \%-0.01 \%)$ [4]. Indeed, Zoledronate infusion for the treatment of metastatic bone disease is frequently associated to BRONJ occurrence, whereas its administration in osteoporosis patients has been shown to substantially reduce fracture risk and increase mineral apposition rate [5, 6]. Up to now, no correlation between BP posology and AFFs has been described yet, but the direct relationship between duration of $\mathrm{BP}$ exposure and risk of developing this pathology is well documented [7].

BPs, being synthetic analogues of inorganic pyrophosphate, can accumulate in bone tissue and be incorporated into bone-resorbing cells. Depending on their chemical structure, they act by inhibiting osteoclast-mediated bone resorption. Once internalized into osteoclasts, non-nitrogen containing BPs are metabolized to non hydrolyzable ATP analogs that interfere with energy metabolism, whereas nitrogen containing BPs affect mevalonate pathway by preventing the prenylation of small GTPase signaling proteins essential for osteoclast morphology and function [8]. Beside this well documented anti-catabolic action on bone tissue, several in vitro evidences suggest that BPs may play a direct role in the process of bone formation as well. In detail, it has been demonstrated that $\mathrm{BP}$ exposure can enhance osteoblast differentiation, proliferation and activity [9-15]. In contrast, it has been suggested that $\mu \mathrm{M}$ or higher concentrations of BPs can inhibit osteoblastogenesis and induce osteoblast apoptosis [16-21]. The use of different experimental models and types of BPs, together with the employ of immortalized cell lines often of murine origin, might be responsible of these conflicting in vitro results on osteoblast metabolism. Therefore, we decided to investigate the effects of nitrogen containing BPs on human primary pre-osteoblasts. Herein, we show that high doses of both BPs exert a cytotoxic effect on osteoblastic cells, while lower doses affect the short-term release of several bone markers and cytokines. Moreover, we also provide evidence of a BP-dependent impairment of bone matrix production, suggesting an overall effect of these compounds on bone quality.

\section{Materials and Methods}

\section{Reagents}

Unless otherwise stated, chemicals and reagents were purchased from Sigma-Aldrich (St. Louis, MO, USA).

\section{Isolation and expansion of human primary pre-osteoblasts}

Bone specimens were obtained from the femoral head of patients subject to total hip replacement surgery, following the procedure approved by IRCCS Istituto Ortopedico Galeazzi (PQ 7.5.125, version 4). For each patient, personal data (age and gender) and medical anamnesis were collected and donors with history of bisphosphonate therapy, both prior and at the time of surgery, were excluded. For pre-osteoblast isolation, trabecular bone was excised from the mid-deep area of the femoral head, selecting harvesting regions distal from the lesions, then minced into fragments with a scalpel and washed with PBS (Phosphate Buffered Saline) several times in order to remove residual adipose and/or hematopoietic tissue. Between washes, samples were vortexed at high speed to further promote the removal of debris and contaminant tissues. Bone chips were then placed, without any step of enzymatic digestion [22, 23], in $60 \mathrm{~mm}$ petri dishes and cultured in high glucose DMEM supplemented with $10 \%$ FBS (Euroclone, Pero, Italy), 2mM L-glutamine, $50 \mathrm{U} / \mathrm{ml}$ penicillin and $50 \mu \mathrm{g} / \mathrm{ml}$ streptomycin at $37^{\circ} \mathrm{C}$ in a humidified atmosphere containing 5\% $\mathrm{CO}_{2}$. Culture media were changed twice a week. When cells outgrown from the explants reached the $90 \%$ confluence, they were detached and sub-cultured every 2 weeks. For the experiments, pre-osteoblasts were employed within the $3^{\text {rd }}$ culture passage. The phenotypic characterization of osteoblastic primary cultures is described in Supplementary material.

\section{Exposure to bisphosphonates}

24 hours after seeding, pre-osteoblasts were exposed to several concentrations of the nitrogen containing BPs Alendronate (Y0001727) and Zoledronate (SML0223) dissolved in culture media. For each assay, control cells grown in the absence of the drugs were cultured in parallel. The wide BP concentration range chosen for the treatments was literature-based and derived from the lack of univocal ex vivo data on BP accumulation in bone.

\section{Cell viability}

$3 \times 10^{3}$ cells $/ \mathrm{cm}^{2}$ were seeded in triplicate on 96-well plates. At day 1, 2, 5 and 9 pre-osteoblasts were treated with a wide range of ALN or ZOL 
concentrations and cell viability was monitored through time as previously described [24]. Briefly, at each time point (day 2, 5, 9 and 12) culture media were replaced with $200 \mu l$ DMEM supplemented with $10 \%$ AlamarBlue ${ }^{\circledR} \quad$ (Thermo Fisher Scientific, Waltham, MA, USA) and cells were incubated for 3.5 hours at $37^{\circ} \mathrm{C}$ in the dark. $100 \mu$ lof supernatants were then transferred to black bottom 96-well plates and fluorescence $(540 \mathrm{~nm}$ excitation $\lambda, 600 \mathrm{~nm}$ emission $\lambda$ ) was read with Wallac Victor II plate reader (Perkin Elmer, Milan, Italy).

\section{ALP activity assessment}

$5 \times 10^{3}$ cells $/ \mathrm{cm}^{2}$ were cultured in 24-wells plates in the presence or absence of BP concentrations spanning from $10^{-13}$ to $10^{-5} \mathrm{M}$. After 14 days, cells were washed in PBS, lysed in 50 $\mu 10.1 \%$ Triton X-100 and the protein content of each sample was quantified through BCA $^{\mathrm{TM}}$ Protein Assay (Thermo Fisher Scientific, Waltham, MA, USA). Alkaline phosphatase enzymatic activity was assessed through a colorimetric assay based on the conversion of p-nitrophenyl phosphate ( $\mathrm{pNPP}$ ) into p-nitrophenol $(\mathrm{pNP})$, following the procedure exhaustively described in [25]. The enzymatic activity (U) was calculated considering the amount of produced $\mathrm{pNP}$ and the reaction time, then normalized to each sample protein content and expressed as ALP specific activity $(\mathrm{U} / \mu \mathrm{g})$.

\section{Calcified extracellular matrix quantification}

$5 \times 10^{3}$ cells $/ \mathrm{cm}^{2}$ were cultured in 24-well plates either in standard conditions or in the presence of $10^{-13}, 10^{-10}$ or $10^{-7} \mathrm{M}$ ALN or ZOL. After 16 days, the deposition of calcified extracellular matrix was quantified following standard procedures [25]. Briefly,samples were fixed with $70 \%$ ethanol and stained with $40 \mathrm{mM}$ Alizarin Red-S. Specific staining was then extracted with $10 \%$ cetylpyridinium chloride in $0.1 \mathrm{M}$ phosphate buffer at $\mathrm{pH} 7.0$ and absorbance was read 550nm with Wallac Victor II plate reader (Perkin Elmer, Milan, Italy).

\section{Primary cell pools}

Cell pools were obtained from three primary populations mixed, at the same subculture passage, following a 1:1:1 ratio. We produced two cell pools with pre-osteoblasts deriving from heterogenous donors, one pool deriving solely from donors younger than $50 \mathrm{y} / \mathrm{o}$ and one from patients older than $60 \mathrm{y} / \mathrm{o}$. Details of the pools are shown in Table 1.

\section{Analysis of released bone biomarkers and cytokines}

Pooled pre-osteoblasts were seeded at a density of $1.5 \times 10^{4} / \mathrm{cm}^{2}$ and treated with $10^{-13}, 10^{-10}$ or $10^{-7} \mathrm{M}$ BPs for 7 days. Conditioned media were collected at day 3 and 7 after treatment, centrifuged at $2000 \mathrm{~g}$ for 5 minutes, then stored at $-20^{\circ} \mathrm{C}$. The MILLIPLEX MAP Human Bone Magnetic Bead Panel-Bone Metabolism Multiplex Assay (HBNMAG-51K, Millipore, Burlington, MA, USA) was customized to contain 8 key bone molecules: DKK-1, IL-6, TNFa, OPG, OPN, SOST, IL-1 $\beta$ and FGF23. Duplicates of conditioned media $(25 \mu \mathrm{l} /$ sample) were read through Bio-Plex Multiplex System (Bio-Rad, Milan, Italy) following standard procedures. IL-6 levels were measured in 1:5 diluted samples. Data analysis was performed with MAGPIX xPONENT 4.2 software (Luminex Corporation, Austin, TX, USA). Levels of secreted RANKL were determined by sandwich enzyme linked immunoassay (ELISA) on culture media in duplicate for each condition, following standard procedures (EK0842, Boster Bio, Pleasanton, CA, USA). Data were analysed with MyAssays software (www.myassays.com).

\section{Analysis of matrix production}

Cells were lysed in $65 \mathrm{mM}$ Tris- $\mathrm{HCl}, 2 \%$ SDS at $\mathrm{pH} 6.8$ supplemented with protease inhibitors. $20 \mu \mathrm{g}$ of whole cell extracts, quantified through BCA $^{\mathrm{TM}}$ Protein Assay (Thermo Fisher Scientific, Waltham, MA, USA), were resolved in SDS-PAGE and transferred to nitrocellulose membranes (GE Healthcare, Milan, Italy). Membranes were probed with antibodies raised against type I Collagen (\#7025, Chondrex, Redmond, WA, USA, dilution 1:5000), Osteopontin (ab8448,Abcam, Hongkong, China, dilution 1:1000) and SPARC (sc-33645, Santa Cruz Biotechnology,CA, USA, dilution 1:3000). $\beta$ Tubulin expression was also revealed (sc-9104, Santa Cruz Biotechnology, CA, USA, dilution 1:1000). Proteins of interest were detected after incubation with appropriate HRP-conjugated secondary antibodies (Santa Cruz Biotechnology, CA, USA, dilution range 1:3000-1:5000) and revealed with LiteAblot ${ }^{\circledR}$ Turbo Extra-Sensitive Chemiluminescent Substrate (Euroclone, Pero, Italy). Images were acquired through ChemiDoc Imaging System ${ }^{\mathrm{TM}}$ and analysed through Image Lab $^{\mathrm{TM}}$ software (Bio-Rad, Milan, Italy).

\section{Statistical analysis}

Unless otherwise stated, data are expressed as mean \pm standard error of the mean (SEM) of at least 3 independent experiments. Statistical analysis was performed by two-tailed unpaired Student's $t$ test using Prism 5 software (GraphPad Software Inc, La Jolla, CA, USA). Differences were considered significant at $\mathrm{p} \leq 0.05$. 


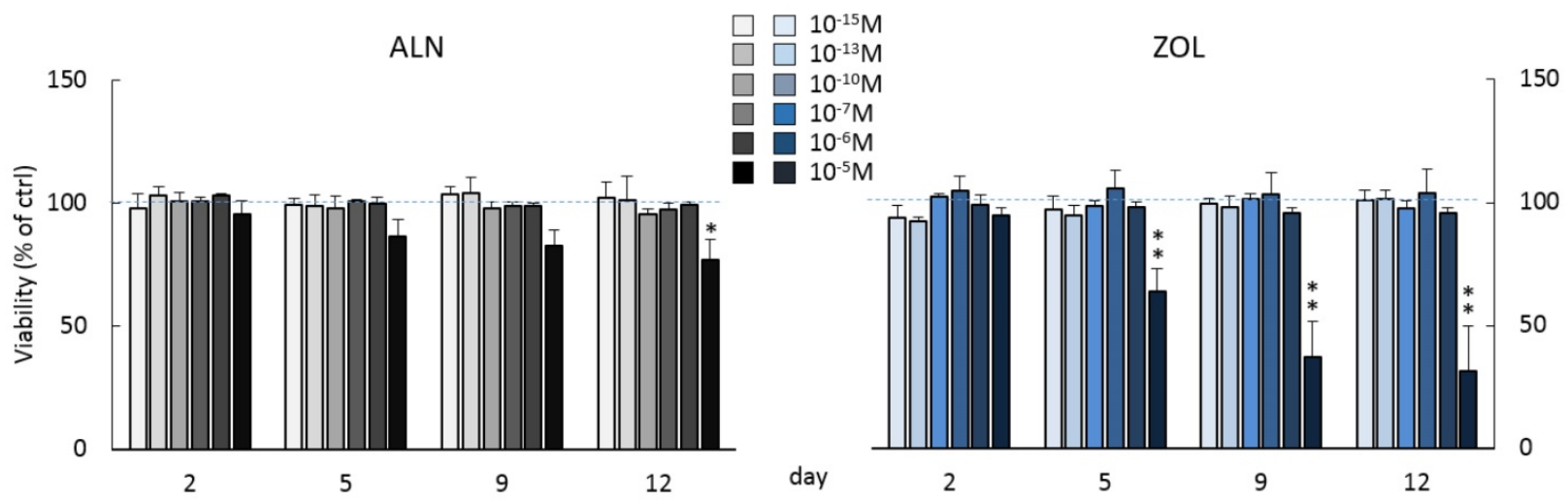

Figure 1. Influence on cell viability of repeated treatments with ALN or ZOL concentrations from 10-15 to 10-5M. Data are represented as relative values setting as $100 \%$ the viability of untreated pre-osteoblasts at every time point (ctrl, blue dashed lines). Values are expressed as mean \pm SEM of at least 3 independent experiments for each condition. Statistical significance versus ctrl is shown as $* \mathrm{p}<0.05$ and $* * \mathrm{p}<0.01$.

\section{Results}

\section{Cytotoxic effect of 10-5M ALN and ZOL on pre-osteoblasts}

Cell viability was monitored for 12 days of chronic BP treatments (concentrations up to $10^{-5} \mathrm{M}$ ). Concentrations below $\mu \mathrm{M}$ did not affect cell viability (Figure 1), while repeated treatments with $10^{-5} \mathrm{M}$ ALN slightly decreased it starting from day 5 . At day 12 , a significant reduction of $-23.2 \pm 8.2 \%$ in respect to untreated pre-osteoblasts was observed. Differently, $\mathrm{ZOL}$ administered at the same concentration exerted a stronger cytotoxic action compared to ALN. A significant reduction of cell viability was detectable starting from day 5 and at the final time point it reached a $-68.7 \pm 18.3 \%$ compared to control cells. Moreover, metabolic and apoptotic stress were perceivable already at day 2 , when pre-osteoblasts acquired a rounder shape and increased vacuole secretion (data not shown). All subsequent experiments were performed using BP concentrations that did not significantly affect cell viability.

\section{BPs influence ALP activity and calcified ECM deposition by pre-osteoblasts}

We investigated the effect of repeated administrations of ALN or ZOL on alkaline phosphatase (ALP) activity and calcified ECM production, both well-recognized markers of osteoblast maturation. As shown in Figure 2A, pre-osteoblast response to ALN stimulus was extremely variable at day 14 , whereas for ZOL a dose response trend was discernible. Indeed, ZOL $\leq 10^{-10} \mathrm{M}$ enhanced ALP activity of about $+25 \%$ in respect to untreated cells, while higher concentrations (ZOL $\geq 10^{-9} \mathrm{M}$ ) slightly decreased it (from $-11 \%$ to $-26 \%)$. As expected, $\mu \mathrm{M}$ concentrations of both compounds inhibited also ALP activity (data not shown). Conversely, ZOL slightly inhibited pre-osteoblast mineralization, while $10^{-13}$ and $10^{-7} \mathrm{M}$ ALN reduced it to a major extent $(-15.7 \pm 7.7 \%$ and $-34.9 \pm 14.3 \%$ in respect to control cells, Figure $2 \mathrm{~B}$ ).

\section{Levels of bone biomarkers and inflammatory cytokines in pre-osteoblast culture medium}

Cultured human primary pre-osteoblasts released detectable levels of IL-6, Osteopontin (OPN), Sclerostin (SOST), Dickkopf-related protein 1 (DKK-1), Osteoprotegerin (OPG), TNF- $\alpha$, IL-1 $\beta$ and RANKL, while FGF-23 was undetectable (Table 2). Interestingly, the low basal levels of TNF- $\alpha$ were increased over time of about $+94 \%$. In addition, also the secretion of IL-1 $\beta$ was mildly upregulated, while the high levels of IL-6, OPN and SOST released up to day 3 seemed to be reduced at day 7. No variation was observed for DKK-1, OPG and RANKL, which maintained an average daily release of $548 \mathrm{pg} / \mathrm{ml}, 1.1$ $\mathrm{ng} / \mathrm{ml}$ and $73.8 \mathrm{pg} / \mathrm{ml}$, respectively.

Table 1. Description of pre-osteoblast pools according to donor characteristics. OA: osteoarthritis.

\begin{tabular}{|c|c|c|c|c|c|}
\hline \multicolumn{3}{|c|}{ Donor characteristics } & \multirow[t]{2}{*}{ Pool } & \multirow{2}{*}{$\begin{array}{l}\text { Culture } \\
\text { passage }\end{array}$} & \multirow{2}{*}{$\begin{array}{l}\text { Mean age } \\
(y / o) \pm S D\end{array}$} \\
\hline Gender & Age (y/o) & Diagnosis & & & \\
\hline$\sigma^{*}$ & 59 & $\mathrm{OA}$ & 1 & $3^{\text {rd }}$ & $56 \pm 12$ \\
\hline$\sigma^{\pi}$ & 66 & $\mathrm{OA}$ & & & \\
\hline 운 & 43 & $\begin{array}{l}\text { Congenital Hip } \\
\text { Dislocation }\end{array}$ & & & \\
\hline$\sigma^{*}$ & 64 & Severe OA & 2 & $3^{\text {rd }}$ & $61 \pm 4$ \\
\hline q & 62 & Femoral Head Necrosis & & & \\
\hline & 56 & $\mathrm{OA}$ & & & \\
\hline$\sigma^{*}$ & 48 & OA & 3 & $2^{\text {nd }}$ & $47 \pm 1$ \\
\hline$\sigma^{*}$ & 48 & OA & & & \\
\hline ९ & 46 & $\mathrm{OA}$ & & & \\
\hline$\sigma^{\prime \prime}$ & 66 & Severe OA & 4 & $2^{\text {nd }}$ & $66 \pm 3$ \\
\hline$\sigma^{*}$ & 69 & $\mathrm{OA}$ & & & \\
\hline @ & 64 & OA & & & \\
\hline
\end{tabular}


A

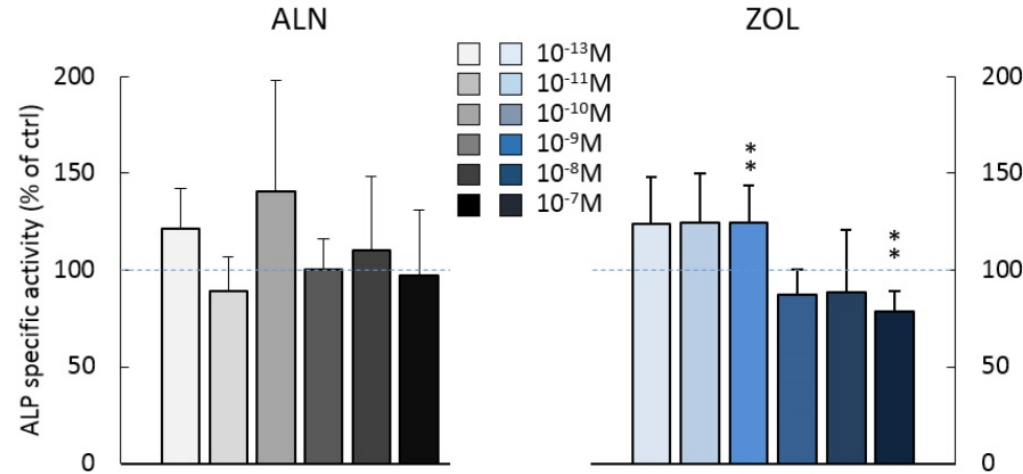

B

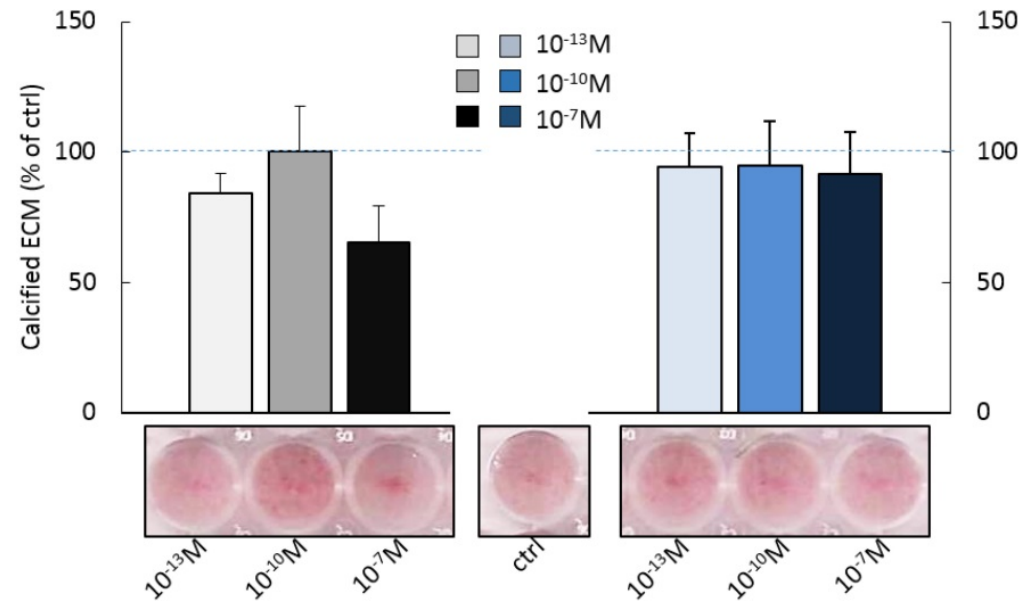

Figure 2. Effect on ALP activity and calcified ECM deposition of repeated treatments with ALN or ZOL concentrations from 10-13 to 10-7M. Data are represented as relative values setting as $100 \%$ the ALP activity (A) or the calcified ECM deposition (B) of untreated pre-osteoblasts (ctrl, blue dashed lines). Representative macrographs of Calcium nodules stained with Alizarin Red-S dye are shown. Results are expressed as mean \pm SEM of at least 3 independent experiments for each condition. Statistical significance versus ctrl is shown as $* * p<0.01$.

Table 2. Bone biomarkers and inflammatory cytokines released by cultured human primary pre-osteoblasts at day 3 and 7. Data are expressed as mean \pm SD of 4 cell pools at day 3 and 3 pools (pool 2-4) at day $7(n=12$ and $n=9$ pre-osteoblast populations respectively). -: non detectable.

\begin{tabular}{lll}
\hline & secreted levels \\
\hline day & $\mathbf{3}$ & 7 \\
IL-6(ng/ml) & $10.7 \pm 1.9$ & $8.2 \pm 1.6$ \\
OPN(ng/ml) & $3.4 \pm 3.8$ & $0.7 \pm 0.1$ \\
SOST $(\mathrm{ng} / \mathrm{ml})$ & $0.36 \pm 0.05$ & $0.24 \pm 0.03$ \\
DKK-1 $(\mathrm{ng} / \mathrm{ml})$ & $1.9 \pm 0.6$ & $2.2 \pm 0.9$ \\
OPG $(\mathrm{ng} / \mathrm{ml})$ & $3.6 \pm 0.9$ & $4.1 \pm 0.2$ \\
TNFa(pg/ml) & $0.09 \pm 0.02$ & $0.21 \pm 0.06$ \\
IL-1 $\beta(p g / m l)$ & $0.16 \pm 0.02$ & $0.22 \pm 0.06$ \\
RANKL $(\mathrm{pg} / \mathrm{ml})$ & $74.6 \pm 1.1$ & $73.1 \pm 0.0$ \\
FGF-23 & - & - \\
\hline
\end{tabular}

\section{BPs influence the release of bone biomarkers and inflammatory cytokines by pre-osteoblasts}

At day 3, the highest doses of both ALN and ZOL down modulated OPN levels (Figure 3A). This mild reduction is maintained up today 7 just with $10^{-7} \mathrm{M}$ ZOL, whereas $10^{-7} \mathrm{M}$ ALN treatment slightly enhanced OPN. The secretion of SOST, DKK-1, TNFa and IL-1 $\beta$ seemed always slightly stimulated at day 3 , although a second BP treatment almost normalized their levels to untreated cells, with only $10^{-7} \mathrm{M}$ ALN still stimulating TNFa release at the later time point (Figure 3D). At day 3, RANKL levels were slightly down modulated and a decreased RANKL/OPG ratio was revealed after $10^{-7} \mathrm{M}$ ALN, $10^{-13}$ and $10^{-10} \mathrm{M}$ ZOL treatment (Figure 3F). In addition, OPG and IL-6 levels were never affected by 7-day treatments (data not shown). In the attempt to disclose differences in OB response depending on donor characteristics, we analyzed our data considering donor age. For most factors, this novel analysis was irrelevant, except for IL-1 $\beta$, whose release displayed an age-related trend in response to $\mathrm{BP}$ administration (Figure 4). In fact, at day 3 all ALN concentrations stimulated preosteoblasts isolated from donors younger than $50 \mathrm{y} / \mathrm{o}$ to secrete IL-1 $\beta$ and a similar trend was observed after ZOL administration. On the contrary, cells harvested from older patients were not affected (data not shown). Unexpectedly, at day 7 both compounds reduced IL-1 $\beta$ release by cells derived from young donors (Figure 4), while cells harvested from elderly patients were either unaffected or slightly stimulated by the highest concentrations (data not shown). 
A

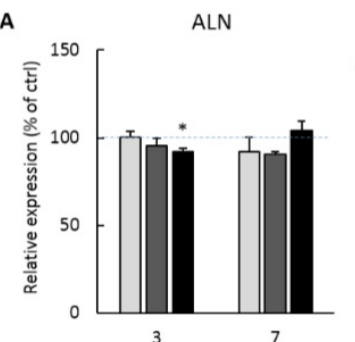

C
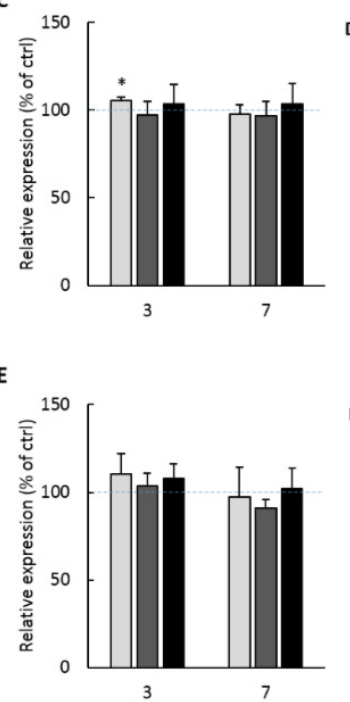

OPN

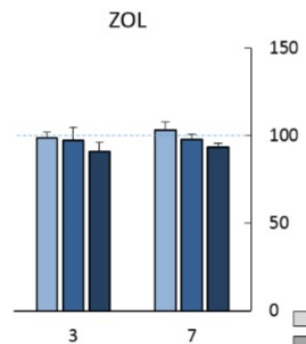

DKK-1

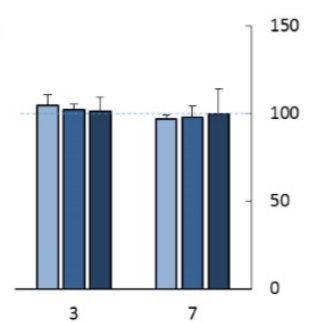

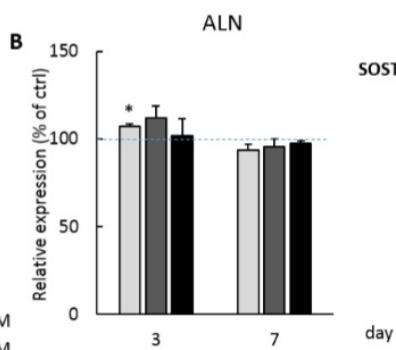
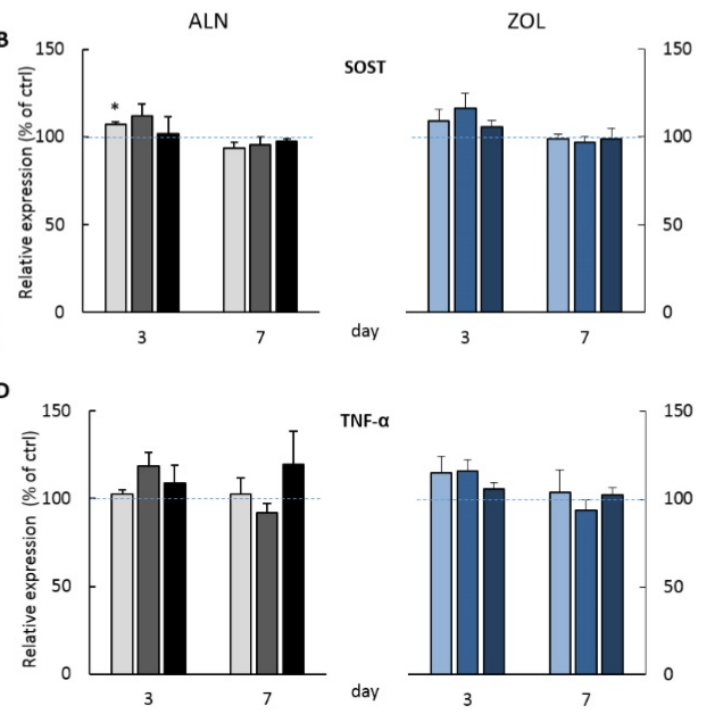

$\mathbf{F}$

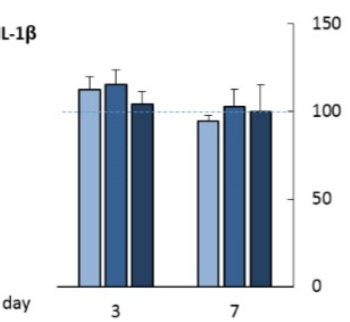

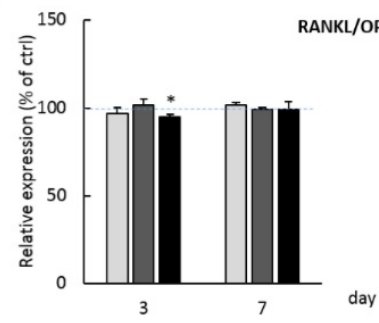

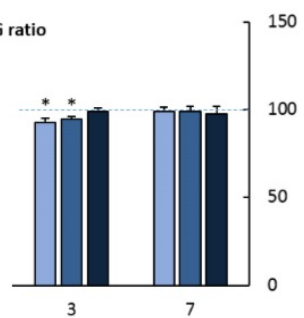

Figure 3. BP effect on protein secretion by pooled human primary pre-osteoblasts. OPN (A) SOST(B) DKK-1 (C) TNFa (D) IL-1 $\beta$ (E) RANKL and OPG (F) release was evaluated at both day 3 and 7 after ALN or ZOL treatment. Data are represented as relative values setting as $100 \%$ the secretion levels of the different analytes by untreated pre-osteoblasts (ctrl, blue dashed lines). Values are expressed as mean \pm SEM of 4 (pool 1-4) and 3 (pool 2-4) independent experiments at each time point ( $n=12$ and $n=9$ cell populations respectively). For each pool, donor characteristics are described in Table 1 . Statistical significance versus ctrl is shown as $* \mathrm{p}<0.05$.

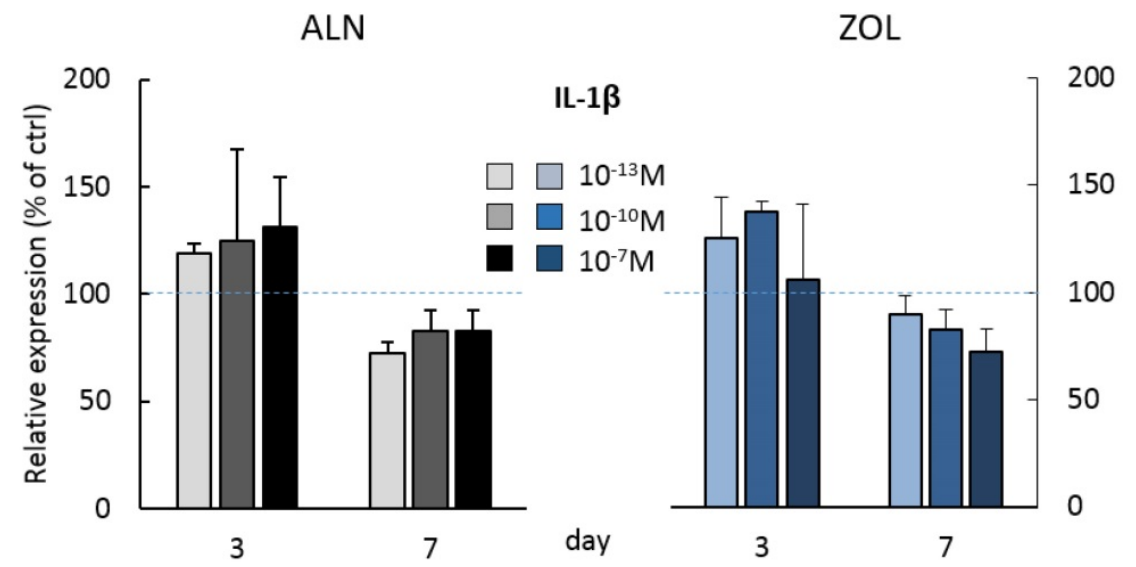

Figure 4. IL-1 $\beta$ secretion by pooled human primary pre-osteoblasts deriving from donors younger than 50 years old (pool 3 , donor characteristics are described in Table 1). IL-1 $\beta$ secretion was evaluated at day 3 and 7 after treatment with ALN and ZOL. Data derive from 3 osteoblastic populations and are expressed as mean \pm SD of technical replicates.

\section{BPs impair the expression of ECM proteins by pre-osteoblasts}

Most concentrations of ALN seemed to reduce both COLL I and OPN expression, whereas only the highest one significantly enhanced SPARC production $(+19.6 \pm 5.8 \%$ in respect to control cells).
Differently, $10^{-7} \mathrm{M}$ ZOL seemed to reduce OPN and COLL I expression (mean decrease of -15 and $-28 \%$, respectively), but all doses favored SPARC increase (higher than $+70 \%$ for $10^{-13}$ and $10^{-10} \mathrm{M}$, around $+34 \%$ for $\left.10^{-7} \mathrm{M}\right)$. 
A

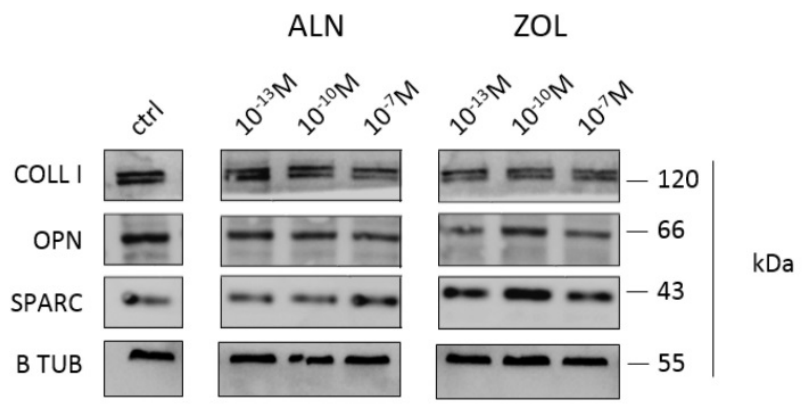

B

ALN

ZOL

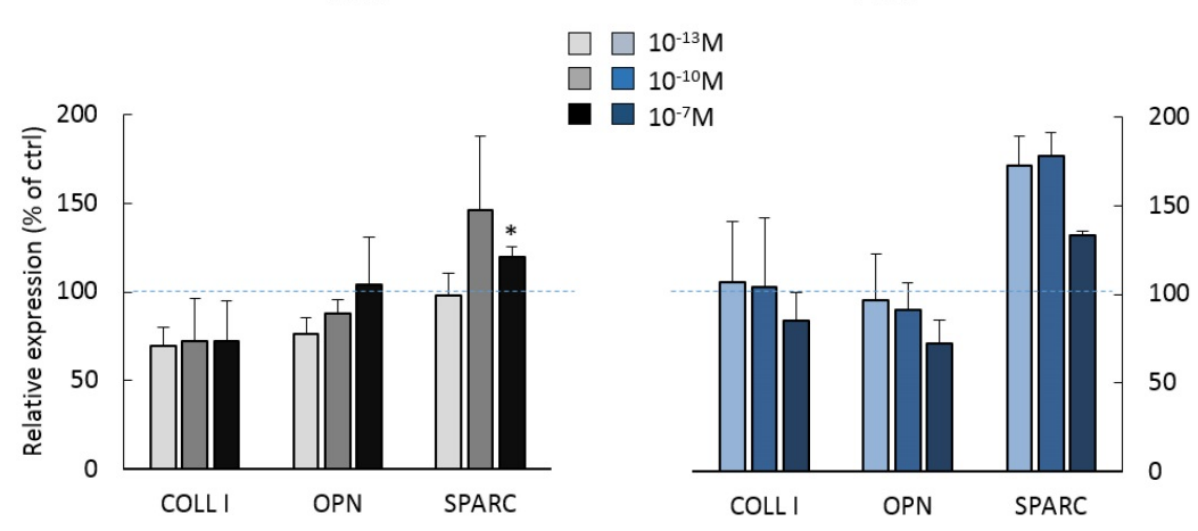

Figure 5. Protein expression by pooled primary human pre-osteoblasts after 7 days of BP treatment. (A) Representative blot with specific bands for COLL I, OPN, SPARC and $\beta$ Tubulin expression. (B) Densitometric evaluation of the expression of ECM proteins by pre-osteoblasts. $\beta$ Tubulin was used as internal control and each analyte was normalized on it. Data are expressed as relative values setting as 100\% the expression of COLL I, OPN or SPARC by untreated pre-osteoblasts (ctrl, blue dashed lines). Results are represented as mean \pm SEM of 3 independent experiments (pool 1, 2 and 4 ) and are obtained from 9 osteoblastic populations. For each pool, donor characteristics are described in Table 1. Statistical significance versus ctrl is shown as ${ }^{*} p<0.05$.

\section{Discussion}

Although it is widely accepted that the pharmacological mechanism of action of BPs mainly relies on the inhibition of osteoclasts, recent evidences suggest that they might directly interfere with osteoblasts, the anabolic counterpart of bone turnover. The process through which BPs are internalized by osteoclasts during the resorptive phase of bone remodeling cycle has been completely unravelled [26], whereas little is known about their incorporation mechanism into non-resorbing cells. In 2008 Coxon et al. were the first to describe the different responses to soluble or mineral-bound BPs by both resorbing and non-resorbing cells. The authors demonstrated that macrophages and osteoblasts grown in monocultures could internalize only limited amounts of drugs from the mineralized matrix. On the contrary, when cells were co-cultured with osteoclasts, the internalization rate was higher, indicating that the release of these molecules from the calcified matrix mediated by osteoclasts can affect neighboring targets [27].

Here we show that high concentrations of both tested drugs strongly reduce pre-osteoblast viability.
Moreover, also alkaline phosphatase activity is decreased by $\mu \mathrm{M}$ doses of BPs (data not show) and $10^{-7} \mathrm{M}$ ZOL, while low concentrations of ZOL slightly increased it. These data corroborate with the hypothesis of a dual nature of BP action on cells belonging to the osteoblastic lineage depending on dosage, recently resumed by Maruotti et al.: an inhibition at concentrations higher than $\mu \mathrm{M}$ and a stimulation at lower doses [28]. However, the pro-osteoblastic/bone-sustaining effect by low BP doses described in literature $[29,30]$, that we observed on ALP activity, in our experimental set up is disproved by analyzing a more mature marker of osteogenesis, the mineralization capacity. Indeed, we gave evidence of an inhibitory trend affecting pre-osteoblast mineralization potential following treatments with both drugs, with ALN exerting a stronger effect.

Since in our hands BPs directly affect metabolism and function of bone-forming cells, we investigated their influence on the release of soluble mediators involved in cell-cell crosstalk within bone milieu. In order to minimize the donor-related variability, we applied a cell pooling strategy, an 
approach quite convenient when working with human primary populations [31, 32]. We are aware that despite this set up allowing reduced variability both within and between experiments, its major disadvantage, beside a diminished statistical power, is the loss of information on the behavior of each individual population [33]. In any case, we believe that data obtained following this approach minimize the impact on the cumulative result of any eventual experimental serendipity or bias.

We gave evidence of a short-term pro-inflammatory and an anti-osteoanabolic effect of ALN and ZOL treatment. In detail, after a single administration of both BPs we observed a positive trend in the secretion of TNFa and IL- $1 \beta$, classically known to inhibit osteoblast differentiation and stimulate bone resorption [34, 35]. This pro-inflammatory phenotype within the bone microenvironment can be interpreted as a positive stimulus to early phase osteogenesis, as in physiological conditions bone regeneration and healing are triggered by the onset of an injury-derived inflammatory reaction [36, 37]. In this perspective, the enhanced early secretion of IL-1 $\beta$ by cells deriving from young patients could fit the physio-pathological context of BP-related skeletal side effects. Indeed, it is well documented that age represents one of the major risk factors associated to BRONJ onset [38]. Our data agree with previous in vitro [39] and ex vivo [40,41] studies that described a correlation between inflammation and nitrogen containing BPs. Here we are the first to show a direct pro-inflammatory action on pre-osteoblasts, since previously it was investigated on immune cells, mainly macrophages $[39,42,43]$. Furthermore, BPs increased the early release of SOST and DKK-1, known inhibitors of osteoblastogenesis acting on Wnt/ $\beta$ catenin pathway [44]. Interestingly, RANKL/OPG ratio was slightly down modulated by the rapid $\mathrm{BP}$ treatment, in contrast to OPG and RANKL levels that were not significantly affected (data not shown). As RANKL promotes osteoclast differentiation and OPG being its decoy receptor, their decreased ratio suggests an inhibitory influence on osteoclastogenesis and osteoclast differentiation. Conversely, in agreement with the hypothesis of a BP-dependent anti-anabolic effect on bone, in treated cells the early secretion of Osteopontin was reduced and a modulation of several intracellular ECM proteins was observed. Indeed, both ALN and $10^{-7} \mathrm{M}$ ZOL slightly inhibited type I Collagen and Osteopontin expression, whereas the production of SPARC seemed to be enhanced, suggesting a drug-dependent modification affecting the quality of the osteoid. In contrast to our results on cell viability and ALP activity, the more potent influence by ALN on the release and/or production of COLL I and OPN raises additional questions on BP mechanism of action. Beside a diverse internalization rate, we suggest that these compounds might interfere on distinct molecular pathways and this aspect will require further investigations. With the perspective of unraveling a direct BP effect on OBs that may explain BRONJ onset, Manzano-Moreno et al. performed short ALN and ZOL treatments on human primary osteoblasts and MG-63 osteosarcoma cell line to evaluate the gene expression of several osteoblast biomarkers [45]. They showed an inhibition of type I Collagen and ALP that partly agrees with our results. Indeed, they found that $10^{-7} \mathrm{M}$ ALN is more potent than ZOL in downregulating COLL I mRNA levels. Moreover, a decreased ALP gene expression in agreement with our reduced activity was observed with $\mu \mathrm{M}$ BP concentrations. Finally, also the lack of modulation of OPG by low BP doses collimates with our data on protein release. Interestingly, they described an increase of OPG mRNA levels in treated MG-63 cells that highlights the possibility of inconsistent results among primary cells and cell lines.

Taken together, our data show that low BP doses act directly on bone-forming cells by increasing the expression of negative bone mediators and impairing ECM quality, suggesting an overall anti-anabolic effect on bone milieu. We believe that the unbalance of bone microarchitecture and cell-cell crosstalk might be related to the development of the drug-related side effects often described at the skeletal level.

\section{Conclusions}

Based on our in vitro results, we hypothesize that BPs may exert an anti-anabolic action within bone microenvironment that could be partially involved in the development of side effects affecting the skeletal system, such as BRONJ or AFFs. We would like to propose that, when osteoblasts are exposed to BPs in pre-pathological conditions, their rapid response may determine an unbalance of the remodelling cycle that, in synergy with other predisposing factors (e.g. bone microdamage, inflammation) and drug-dependent effects (e.g. soft tissue toxicity, inhibition of angiogenesis), may concur to the pathology onset.

\section{Supplementary Material}

Supplementary method and figure.

http://www.medsci.org/v16p0023s1.pdf

\section{Abbreviations}

ALN: Alendronate; $\operatorname{AFF}(\mathrm{s})$ : atypical femoral fracture(s); $\quad \mathrm{BP}(\mathrm{s})$ : Bisphosphonate(s); BRONJ: Bisphosphonate-Related Osteonecrosis of the Jaw; 
COLL I: Type I Collagen; DKK-1: Dickkopf-related protein 1; DT: Doubling time; ECM: Extracellular matrix; IL-1 $\beta$ : Interleukin 1 $\beta$; IL-6: Interleukin 6; OA: Osteoarthritis; OB(s): Pre-osteoblast(s); OC: Osteocalcin; OPG: Osteoprotegerin; OPN: Osteopontin; PBS: Phosphate Buffered Saline; SOST: Sclerostin; SPARC: Osteonectin; TNFa: Tumor Necrosis Factor a; ZOL: Zoledronate.

\section{Acknowledgments}

This study was supported by IRCCS Istituto Ortopedico Galeazzi (RC L2033) and Department of Biomedical Surgical and Dental Sciences, University of Milan (14-2-3017000-511).

\section{Competing Interests}

The authors have declared that no competing interest exists.

\section{References}

1. Vargas-Franco JW, Castaneda B, Redini F, Gomez DF, Heymann D, Lezot F. Paradoxical side effects of bisphosphonates on the skeleton: What do we know and what can we do? J Cell Physiol 2018; 233: 5696-5715.

2. Gedmintas L, Solomon DH, Kim SC. Bisphosphonates and risk of subtrochanteric, femoral shaft, and atypical femur fracture: a systematic review and meta-analysis. J Bone Miner Res 2013; 28: 1729-1737.

3. Compston J. Pathophysiology of atypical femoral fractures and osteonecrosis of the jaw. Osteoporos Int 2011; 22: 2951-2961.

4. Khan AA, Morrison A, Kendler DL, Rizzoli R, Hanley DA, Felsenberg D, et al. Case-Based Review of Osteonecrosis of the Jaw (ONJ) and Application of the International Recommendations for Management From the International Task Force on ONJ. J Clin Densitom 2017; 20: $8-24$.

5. Recker RR, Delmas PD, Halse J, Reid IR, Boonen S, Garcia-Hernandez PA, et al. Effects of intravenous zoledronic acid once yearly on bone remodeling and bone structure. J Bone Miner Res 2008; 23: 6-16.

6. Black DM, Delmas PD, Eastell R, Reid IR, Boonen S, Cauley JA, et al. Once-yearly zoledronic acid for treatment of postmenopausal osteoporosis. N Engl J Med 2007; 356: 1809-1822.

7. Starr J, Tay YKD, Shane E. Current Understanding of Epidemiology, Pathophysiology, and Management of Atypical Femur Fractures. Curr Osteoporos Rep 2018; 16: 519-529.

8. Drake MT, Clarke BL, Khosla S. Bisphosphonates: mechanism of action and role in clinical practice. Mayo Clin Proc 2008; 83: 1032-1045.

9. Reinholz GG, Getz B, Pederson L, Sanders ES, Subramaniam M, Ingle JN, et al. Bisphosphonates directly regulate cell proliferation, differentiation, and gene expression in human osteoblasts. Cancer Res 2000; 60: 6001-6007.

10. Im GI, Qureshi SA, Kenney J, Rubash HE, Shanbhag AS. Osteoblast proliferation and maturation by bisphosphonates. Biomaterials 2004; 25: 4105-4115.

11. von Knoch F, Jaquiery C, Kowalsky M, Schaeren S, Alabre C, Martin I, et al. Effects of bisphosphonates on proliferation and osteoblast differentiation of human bone marrow stromal cells. Biomaterials 2005; 26: 6941-6949.

12. Wang CZ, Chen SM, Chen CH, Wang CK, Wang GJ, Chang JK, et al. The effect of the local delivery of alendronate on human adipose-derived stem cell-based bone regeneration. Biomaterials 2010; 31: 8674-8683.

13. Casado-Diaz A, Santiago-Mora R, Dorado G, Quesada-Gomez JM. Risedronate positively affects osteogenic differentiation of human mesenchymal stromal cells. Arch Med Res 2013; 44: 325-334.

14. Imai $\mathrm{Y}$, Hasegawa $\mathrm{T}$, Takeda $\mathrm{D}$, Akashi M, Komori T. The osteogenic activity of human mandibular fracture haematoma-derived progenitor cells is affected by bisphosphonate in vitro. Int J Oral Maxillofac Surg 2015; 44: 412-416.

15. Kaiser T, Teufel I, Geiger K, Vater Y, Aicher WK, Klein G, et al. Bisphosphonates modulate vital functions of human osteoblasts and affect their interactions with breast cancer cells. Breast Cancer Res Treat 2013; 140: 35-48.
16. Garcia-Moreno C, Serrano $S$, Nacher M, Farre M, Diez A, Marinoso ML, et al. Effect of alendronate on cultured normal human osteoblasts. Bone 1998; 22: 233-239.

17. Naidu A, Dechow PC, Spears R, Wright JM, Kessler HP, Opperman LA. The effects of bisphosphonates on osteoblasts in vitro. Oral Surg Oral Med Oral Pathol Oral Radiol Endod 2008; 106: 5-13.

18. Idris AI, Rojas J, Greig IR, Van't Hof RJ, Ralston SH. Aminobisphosphonates cause osteoblast apoptosis and inhibit bone nodule formation in vitro. Calcif Tissue Int 2008; 82: 191-201.

19. Patntirapong $S$, Singhatanadgit $W$, Chanruangvanit $C$, Lavanrattanakul $\mathrm{K}$, Satravaha Y. Zoledronic acid suppresses mineralization through direct cytotoxicity and osteoblast differentiation inhibition. J Oral Pathol Med 2012; 41: 713-720.

20. Walter C, Pabst AM, Ziebart T. Effects of a low-level diode laser on oral keratinocytes, oral fibroblasts, endothelial cells and osteoblasts incubated with bisphosphonates: An in vitro study. Biomed Rep 2015; 3: 14-18.

21. Huang X, Huang S, Guo F, Xu F, Cheng P, Ye Y, et al. Dose-dependent inhibitory effects of zoledronic acid on osteoblast viability and function in vitro. Mol Med Rep 2016; 13: 613-622.

22. Chen SM, Peng YJ, Wang CC, Su SL, Salter DM, Lee HS. Dexamethasone Down-regulates Osteocalcin in Bone Cells through Leptin Pathway. Int J Med Sci 2018; 15: 507-516.

23. Dillon JP, Waring-Green VJ, Taylor AM, Wilson PJ, Birch M, Gartland A, et al. Primary human osteoblast cultures. Methods Mol Biol 2012; 816: 3-18.

24. Giannasi C, Pagni G, Polenghi C, Niada S, Manfredi B, Brini AT, et al. Impact of Dental Implant Surface Modifications on Adhesion and Proliferation of Primary Human Gingival Keratinocytes and Progenitor Cells. Int J Periodontics Restorative Dent 2018; 38: 127-135.

25. Niada S, Giannasi C, Ferreira LM, Milani A, Arrigoni E, Brini AT. 17beta-estradiol differently affects osteogenic differentiation of mesenchymal stem/stromal cells from adipose tissue and bone marrow. Differentiation 2016

26. Thompson K, Rogers MJ, Coxon FP, Crockett JC. Cytosolic entry of bisphosphonate drugs requires acidification of vesicles after fluid-phase endocytosis. Mol Pharmacol 2006; 69: 1624-1632.

27. Coxon FP, Thompson K, Roelofs AJ, Ebetino FH, Rogers MJ. Visualizing mineral binding and uptake of bisphosphonate by osteoclasts and non-resorbing cells. Bone 2008; 42: 848-860.

28. Maruotti N, Corrado A, Neve A, Cantatore FP. Bisphosphonates: effects on osteoblast. Eur J Clin Pharmacol 2012; 68: 1013-1018.

29. Ebert R, Zeck S, Krug R, Meissner-Weigl J, Schneider D, Seefried L, et al. Pulse treatment with zoledronic acid causes sustained commitment of bone marrow derived mesenchymal stem cells for osteogenic differentiation. Bone 2009; 44: 858-864.

30. Adam C, Gluck L, Ebert R, Goebeler M, Jakob F, Schmidt M. The MEK5/ERK5 mitogen-activated protein kinase cascade is an effector pathway of bone-sustaining bisphosphonates that regulates osteogenic differentiation and mineralization. Bone 2018; 111: 49-58.

31. Ketterl N, Brachtl G, Schuh C, Bieback K, Schallmoser K, Reinisch A, et al. A robust potency assay highlights significant donor variation of human mesenchymal stem/progenitor cell immune modulatory capacity and extended radio-resistance. Stem Cell Res Ther 2015; 6: 236.

32. Nieto-Nicolau N, Martinez-Conesa EM, Casaroli-Marano RP. Limbal Stem Cells from Aged Donors Are a Suitable Source for Clinical Application. Stem Cells Int 2016; 2016: 3032128.

33. Stoddart MJ, Richards RG, Alini M. In vitro experiments with primary mammalian cells: to pool or not to pool? Eur Cell Mater Scotland 2012:i-ii.

34. Abbas $\mathrm{S}$, Zhang $\mathrm{YH}$, Clohisy JC, Abu-Amer Y. Tumor necrosis factor-alpha inhibits pre-osteoblast differentiation through its type-1 receptor. Cytokine 2003; 22: 33-41.

35. Kwan Tat S, Padrines $M$, Theoleyre $S$, Heymann D, Fortun $Y$. IL-6, RANKL, TNF-alpha/IL-1: interrelations in bone resorption pathophysiology. Cytokine Growth Factor Rev 2004; 15: 49-60.

36. Ebert R, Benisch P, Krug M, Zeck S, Meissner-Weigl J, Steinert A, et al. Acute phase serum amyloid A induces proinflammatory cytokines and mineralization via toll-like receptor 4 in mesenchymal stem cells. Stem Cell Res 2015; 15: 231-239.

37. Kolar P, Schmidt-Bleek K, Schell H, Gaber T, Toben D, Schmidmaier G, et al. The early fracture hematoma and its potential role in fracture healing. Tissue Eng Part B Rev 2010; 16: 427-434.

38. Ruggiero SL, Dodson TB, Fantasia J, Goodday R, Aghaloo T, Mehrotra B, et al. American Association of Oral and Maxillofacial Surgeons position paper on medication-related osteonecrosis of the jaw--2014 update. J Oral Maxillofac Surg 2014; 72: 1938-1956.

39. Muratsu D, Yoshiga D, Taketomi T, Onimura T, Seki Y, Matsumoto A, et al. Zoledronic acid enhances lipopolysaccharide-stimulated 
proinflammatory reactions through controlled expression of SOCS1 in macrophages. PLoS One 2013; 8: e67906.

40. Norton JT, Hayashi T, Crain B, Cho JS, Miller LS, Corr M, et al. Cutting edge: nitrogen bisphosphonate-induced inflammation is dependent upon mast cells and IL-1. J Immunol 2012; 188: 2977-2980.

41. de Barros Silva PG, de Oliveira CC, Brizeno L, Wong D, Lima Junior R, Goncalves RP, et al. Immune cellular profile of bisphosphonate-related osteonecrosis of the jaw. Oral Dis 2016; 22: 649-657.

42. Santini D, Fratto ME, Vincenzi B, La Cesa A, Dianzani C, Tonini G. Bisphosphonate effects in cancer and inflammatory diseases: in vitro and in vivo modulation of cytokine activities. BioDrugs 2004; 18: 269-278.

43. Monkkonen J, Simila J, Rogers MJ. Effects of tiludronate and ibandronate on the secretion of proinflammatory cytokines and nitric oxide from macrophages in vitro. Life Sci 1998; 62: P195-102.

44. Baron R, Kneissel M. WNT signaling in bone homeostasis and disease: from human mutations to treatments. Nat Med 2013; 19: 179-192.

45. Manzano-Moreno FJ, Ramos-Torrecillas J, Melguizo-Rodriguez L, Illescas-Montes R, Ruiz C, Garcia-Martinez O. Bisphosphonate Modulation of the Gene Expression of Different Markers Involved in Osteoblast Physiology: Possible Implications in Bisphosphonate-Related Osteonecrosis of the Jaw. Int J Med Sci 2018; 15: 359-367. 